\title{
Acoustic devices for breathing investigations
}

\author{
Semyon Shkundin ${ }^{1, *}$ and Valentina Rumyantseva ${ }^{2, * *}$ \\ ${ }^{1}$ MISIS National University of Science and Technology \\ ${ }^{2}$ Bauman Moscow State Technical University
}

\begin{abstract}
The problem of spirometry control in medicine and medical engineering still remains urgent. The necessity of getting more and more information from spirometry investigations imposes more and more stringent requirements for spirometers, volumeters and bodypletizmographs, first of all, to the primary spiroflow transdusers. Practice shows that these requirements cannot be met by improving devices which use conventional spirometric principles. The new acoustic means for pulsating air-gas flow rate measurement has been created in Russia and is described in the paper.

The main feature is special air-metric channel, supplied with ceramics electroacoustic transducers. The principle of its operation is based on the dependence of the acoustic vibrations arriving at the receiver velocity upon the air-gas flow rate. The device does not disturb the air dynamic structure of the flow, has no inertia or moving elements and unsurpassed sensitivity and precision.
\end{abstract}

\section{Introduction}

Currently, there is an increase of the number of persons registered for the first time in connection with respiratory diseases. The increased incidence is largely due to the progressive deterioration of the ecological state of the environment. The growth of cities and industrial areas cause pollution in local streams, rivers and airspace. High morbidity associated with the respiratory system, is a priority problem in health care and medical science [1].

The main causes of COPD (chronic obstructive pulmonary disease) are smoking, industrial dust and gases, adverse environmental conditions. The danger of this disease lies in the fact that the formation of irreversible effects starts long before the first symptoms. Due to the fact that COPD develops slowly, it is frequently diagnosed in people aged 40 years and older. Experts in the field of health care strongly recommend that everyone go through the simple procedure - spirometry. If the result shows the lung disfunction, you should consult with your doctor about methods of prevention and treatment of chronic obstructive pulmonary disease. The main requirements to modern spirometers and methodics they have to support are low inertia, high precision and low flow resistance. The measurement of volumes and characteristics of the exhaled and inhaled air can be implemented in different ways, based on variety of methods.

Acoustic method for the rate of air flow measurement has several advantages comparatively to other methods: the precision, reliability, low inertia, no moving parts and obstacles

\footnotetext{
*e-mail: shkundin@mail.ru

**e-mail: valar@bmstu.ru
} 


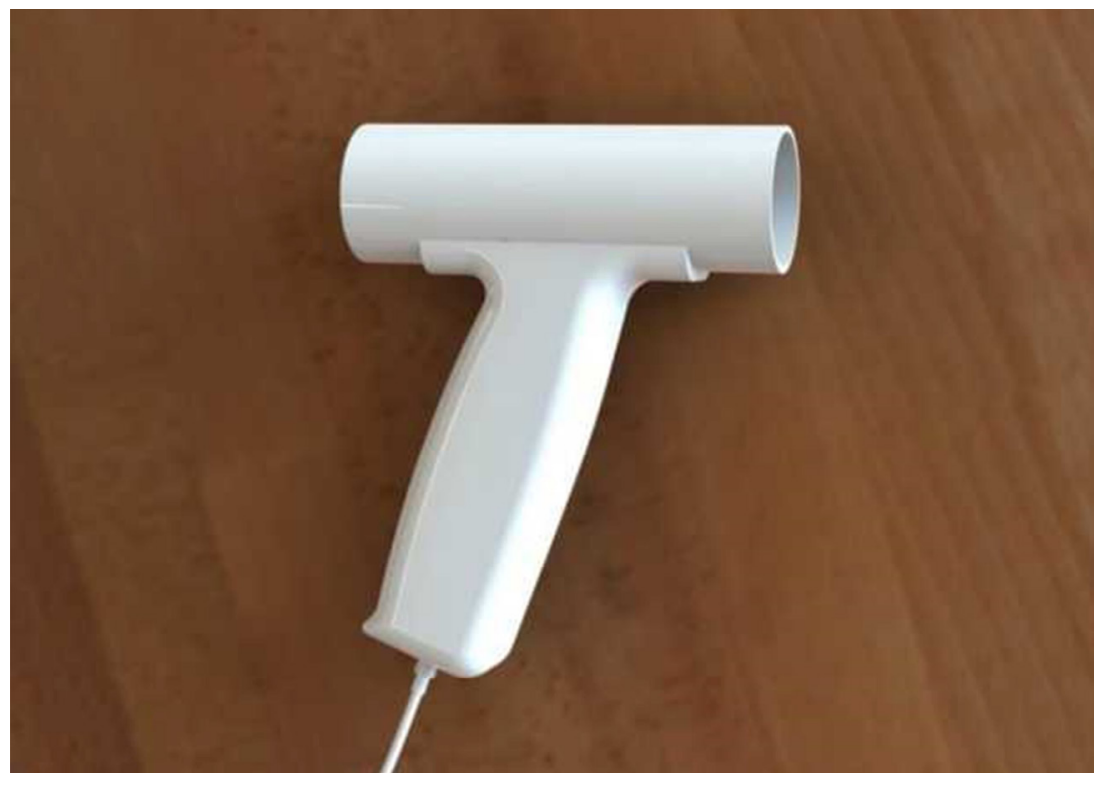

Figure 1. Acoustic sensor spirometer

for the air flow in the sensor and relatively low cost. The image of the acoustic spirometer is shown in Fig. 1.

Traditionally, the disadvantages of the technique is the dependence of the readings on the speed of sound. However, currently, with the development of computer and microprocessor technologies it became possible to process the sensor information and eliminate the speed of sound error at each step of the measurement with the help of specially developed algorithms. Therefore, the development of such algorithms is an actual scientific-technical problem. The creation of them requires a description of the velocity field and the aeroacoustic interaction in the channel that makes the tasks of modeling and description of the physical processes underlying the acoustic measurement method of flow velocity implementation.

In this simulation, the authors solved a number of aerodynamics and acoustics problems, allowing to model and then, as a result of researches, to create new plague level devices with unmatched performance.

\section{Modeling acoustic interaction with a homogeneous flow}

Acoustic spirometer needs to have such properties as reliability, low inertia, accuracy, a wide dynamic range. At the National Investigation Research University under the leadership of prof. Shkundin S. Z. the number of spirometric acoustics devices has been developed [2].

The acoustic sensor of the spirometer is a cylindrical wave guide-air duct with builtin wall piezoelectric transducers, which alternately in time, are actuating as transmitter or receiver of acoustic waves. According to the phase difference of acoustic signals, propagating along and against the air flow, one can determine airspeed. This can be shown in a simple model of propagation of a plane wave in a homogeneous flow in unlimited space.

However, in reality this is not so simple. Acoustic field in the spirometric channel forms a complex wave pattern that can be approximately described with the help of basic system of 
aerohydrodynamics equations with boundary conditions defined by parameters of the waveguide and the used approximation [3]. In the case of homogeneous flow the acoustic field potential and the system of hydrodynamics can be reduced to the wave equation (1) with respect to the acoustic potential $[4,5]$.

We introduce a cylindrical coordinate system z-axis which is the axis of symmetry of the cylindrical wave guide-air duct (Fig. 2). We neglect the reflections of the acoustic waves from the open ends of the waveguide, using the approximation of a cylindrical waveguide with infinite lengths.

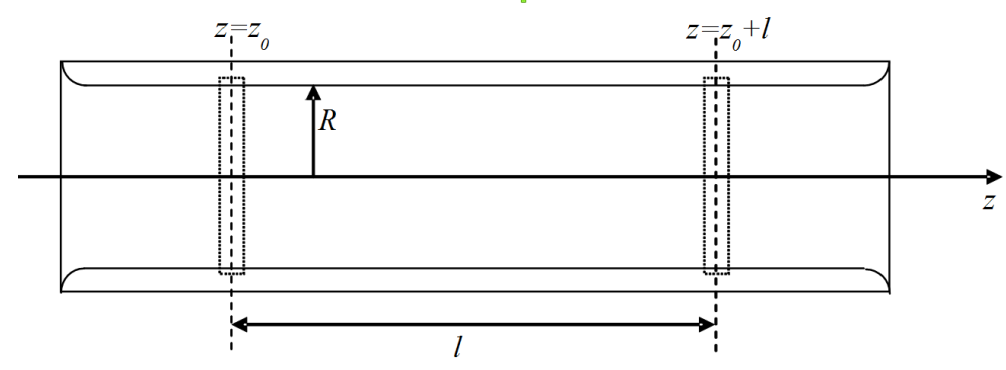

Figure 2. The drawing of the acoustic spirometer sensor

\subsection{An infinite cylindrical waveguide}

Rationale for acoustic measurement of air velocity methods was given by Skundin S. Z. [2] using the results of theoretical work [6]. He has solved the problem of acoustic waves propagation in infinite cylindrical waveguide with perfectly rigid walls with an annular source of acoustic oscillations in the presence of uniform flow. The system of hydrodynamics equations for an ideal environment allows to get the wave equation in a uniform form.

$$
-\frac{1}{c^{2}} \frac{\partial^{2} \varphi}{\partial t^{2}}-\frac{2 M}{c} \frac{\partial^{2} \varphi}{\partial z \partial t}+\Delta \varphi-M^{2} \frac{\partial^{2} \varphi}{\partial z^{2}}=0
$$

Here $\varphi(r, z, t)=\phi(r, z) \dot{e}^{\mathrm{i} \omega t}$ - acoustic potential $\left(\vec{v}^{\prime}=\nabla \varphi, p^{\prime}=-\rho_{0} \frac{\partial \varphi}{\partial t}-\rho_{0}(V \nabla) \varphi\right), c$ - the adiabatic speed of sound, $M$ - Mach number. Further $\rho(\vec{r}, t), \vec{v}(\vec{r}, t), p(\vec{r}, t)$ are density, velocity, pressure, i.e. characteristics of the environment, as a function of position $\vec{r}$ and time $t$. They are represented as the sum of a constant and a variable component: $p=p_{0}+p^{\prime}$, $\rho=\rho_{0}+\rho^{c}, \vec{v}=\vec{v}^{\natural}+\vec{V}, \Delta=\frac{1}{r} \frac{\partial}{\partial r}\left(r \frac{\partial}{\partial r}\right)+\frac{\partial^{2}}{\partial z^{2}}$ - Laplace operator in cylindrical coordinates for axial symmetry. The solution of the wave equation (1) with boundary conditions (2) can be presented as (3):

$$
\begin{gathered}
\left.\frac{\partial \varphi}{\partial r}\right|_{r=R}=\left\{\begin{array}{c}
V_{0} e^{\mathrm{i} \omega t},\left|z-z_{0}\right| \leq h / 2 \\
0,\left|z-z_{0}\right|>h / 2
\end{array}\right. \\
\phi(r, z)=\sum_{n=0}^{\infty} \frac{-2 V_{0} \mathrm{i} \sin \left(\frac{k M \mp \sigma_{n}}{\beta^{2}} h\right) J_{0}\left(\frac{\mu_{n} r}{R}\right) \exp \left(\mathrm{i} \frac{k M \mp \sigma_{n}}{\beta^{2}}\left(z-z_{0}\right)\right)}{\frac{k M \mp \sigma_{n}}{\beta^{2}} R \sigma_{n} J_{2}\left(\mu_{n}\right) \theta_{n}}
\end{gathered}
$$

where $\sigma_{n}=\left\{\begin{array}{c}\sqrt{k^{2}-\beta^{2} \frac{\mu_{n}^{2}}{R^{2}}} n<M_{2} \\ -\mathrm{i} \sqrt{\beta^{2} \frac{\mu_{n}^{2}}{R^{2}}-k^{2}} \\ n<M_{2}\end{array}, \theta_{n}=\left\{\begin{array}{cc}2, & n=0 \\ 1 & n \neq 0\end{array}, \beta=\sqrt{1-M^{2}}, k=\frac{\omega}{c}\right.\right.$ 
Conditions (2) correspond to the infinite waveguide with perfectly rigid walls and a source of acoustic oscillations in the form of a ring (ring height is $h$ ) located at $z=z_{0}$ : Decision: $\varphi_{ \pm}(r, z, t)=\left\{\begin{array}{l}\phi_{+}(r, z) \exp (\mathrm{i} \omega t) z-z_{0} \geq 0 \\ \phi_{-}(r, z) \exp (\mathrm{i} \omega t) z-z_{0}<0\end{array}\right.$, was obtained as a sum of normal modes - harmonic components propagating in the waveguide $\left(n=0,1 \ldots M_{2}\right)$ and exponentially damped $\left(n=M_{2}+1, M_{2}+1 \ldots\right) \cdot M_{2}+1$ is the number of propagating modes.

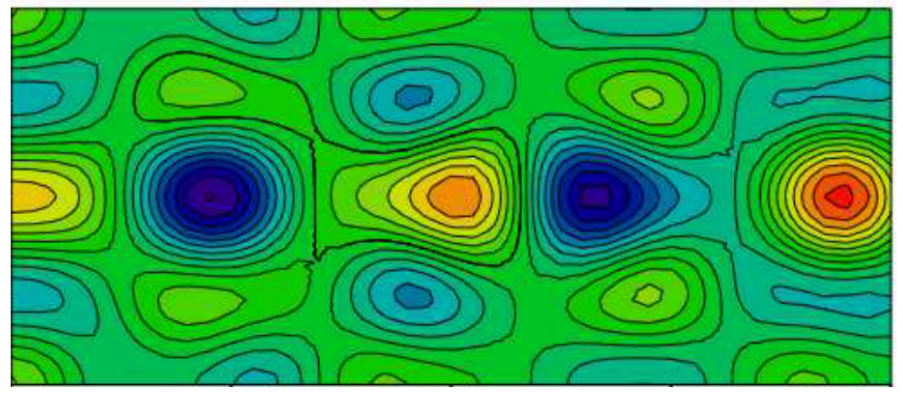

Figure 3. The acoustic potential at a fixed time. A fragment of the infinite waveguide

\subsection{A cylindrical waveguide with finite length}

Acoustic wave propagating from the source along the axis of the waveguide, is being reflected by its edges [7]. The reflected wave, superimposed on the primary wave form a standing wave. Thus, the wave field in a waveguide of finite length is the sum of running from source, and standing waves. As an informative parameter of acoustic anemometer is a phase, it is important to track the impact of reflections on the phase of the received signal.

The problem of propagation of acoustic waves in a uniform flow in a waveguide of finite length was solved by S.Z. Shkundin using the method of Wiener-Hopf [8]. The walls of the waveguide were assumed to be infinitely thin. For getting of the solution the results obtained by Johnson and Ogimoto [9] for an elementary source were used. In our case, the source was modeled as a synchronously oscillating ring. The same problem was solved later by O. A. Kremleva [10] with the help of generalized scattering matrix method, similarly with works [11], where we consider the case of the medium without flow. The coefficients of reflection and transformation at the edges of the waveguide in this case were calculated by the method of crosslinking.

The problem of wave propagation in a channel with walls of finite length and thickness was solved by Rumyantseva V.A. [12] with the help of the generalized scattering matrix method. The coefficients of reflection and transformation were calculated using Wiener-Hopf method, as the description of the waves propagation in semi-infinite waveguide,similarly with the work of Ando [13], where the problem is solved for semi-infinite circular pipe of certain wall thickness without flow.

Figure 4 shows graphs of acoustic oscillations amplitude distribution along the axis of the anemometer channel. The dotted line is the graphics for the waveguide with infinitely thin walls, solid for a waveguide with walls of finite thickness, with rectangular flanges. The vertical axis represents the pressure in relative units (relative to the pressure amplitude at the emitter). The graphics are designed for the following values of parameters [14]: $a_{1}=1.7$ $\mathrm{cm}$ (external radius) $R=a_{2}=1.4 \mathrm{~cm}$ (inner radius) $l=10 \mathrm{~cm}$ (base length), $z_{0}=-7 \mathrm{~cm}$, $\omega=2 \pi f, f=30 \mathrm{kHz}, c=343 \mathrm{~m} / \mathrm{s}, h=3.5 \mathrm{~mm}$. 


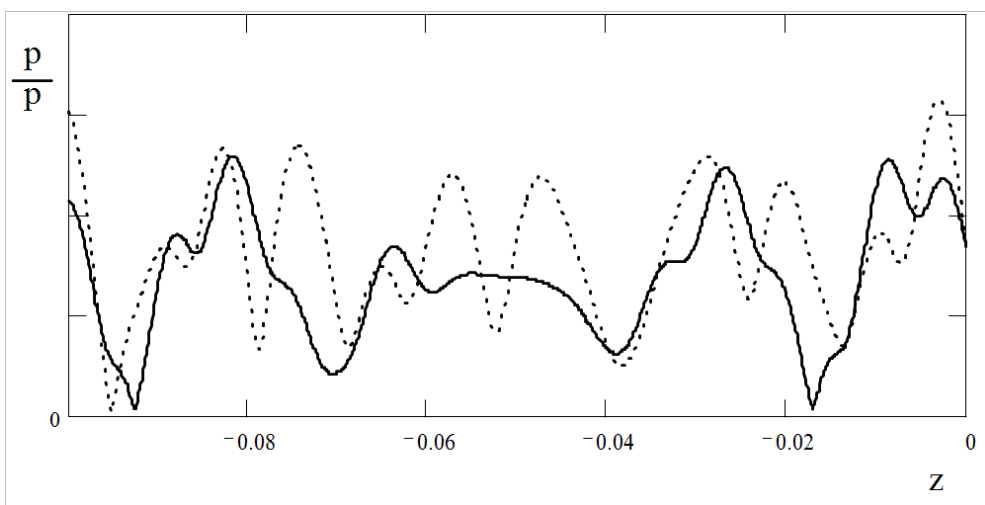

Figure 4. The amplitude of the acoustic pressure on the wall of the waveguide

\subsection{Simulation of the acoustic anemometer sensor with pulse mode radiation}

From the graph shown in figure 4 it can be seen that the reflections have a significant impact on the field in the waveguide, and the case with small but finite thickness walls differs from the case of infinitely thin walls significantly. It turns out that the field in the waveguide is the superposition of many comparable components, so consideration of all the factors significantly affecting the phase of the received signal, in the theoretical model is rather complicated. Therefore an instrument that uses a pulsed irradiation mode is developed. This method allows to separate in time different modes and reflection and in a certain time rangework only with one of the normal modes.

Theoretical justification and calculation of parameters of such devices was carried out by Buyanov S. I. [15]. Model of the propagation of acoustic pulse in a cylindrical waveguide was obtained using the Fourier method. The simulation result of the received signal in a pulsed mode, taking into account the double reflection is shown in figure 5. In this model the walls of the waveguide were considered to be infinitely thin and hard. The remaining parameters correspond to the real sensor.

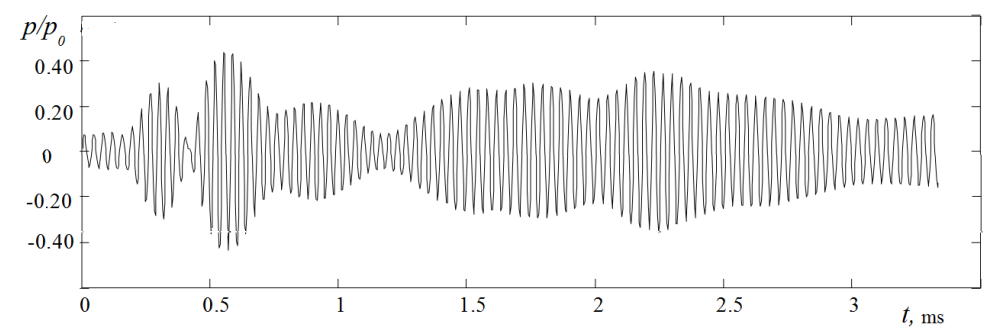

Figure 5. The propagation of a pulse and two reflections in the anemometer channel

\section{Conclusion}

The results of aeroacoustic interaction in spirometric channel modeling help to substitute the phase method for the speed of the air flow measurment, and also to consider the impact of 
the most significant factors on the device operation. The use of theoretical models in the development of new diagnostics methods, allows to decrease various errors i.e. to improve the accuracy of exhaled and inhaled volumes measurement and consequently to improve the diagnostics of pulmonary diseases with the help of spirometry.

\section{References}

[1] M. Arkhipov, A. Leskov, V. Golovin,Y. Gercik, L. Kocherevskaya Advances in Intelligent Systems and Computing, 540, 2017, pp 499-506, 25th International Conference on Robotics in Alpe-Adria-Danube Region, RAAD 2016; Belgrade; Serbia; 30 June 2016 - 2 July 2016;

[2] S.Z. Shkundin, V.B. Lashin Metrologiya [Metrology] 7, pp.39-43 (1990) (in Russian)

[3] A.A. Pozhalostin, D.A. Goncharov, Russian Aeronautics 58, Issue 1, pp. 37-41 (2015)

[4] V.A. Kovalev, E.V. Murashkin, Y.N. Radayev Journal of Physics: Conference Series 991, Issue 1, Nom. 012047 (2017)

[5] E.V. Murashkin, Y.N. Radayev Journal of Physics: Conference Series Vol. 937, Issue 1, Nom. 012031 (2017)

[6] A.D. Lapin Akustiko-aehrodinamicheskie issledovaniya: sbornik. pod red. RimskogoKorsakova.[ Acoustic-aerodynamic studies: a collection. ed. by Rimsky-Korsakov.] Moscow.: 1975. pp. 57-60. (in Russian)

[7] N. Kanev, INTER-NOISE 2017 - 46th International Congress and Exposition on Noise Control Engineering: Taming Noise and Moving Quiet Volume 2017-January;

[8] S.Z. Shkundin, A.M. Bondarev, A.A. Lihachev Gornyj zhurnal. Izv .VUZov, [Mining journal. Proceedings of the Universities] 9 (1987) (in Russian)

[9] K.Ogimoto. UTIAS Report, 231, 1978.

[10] O.A. Kremleva, S.Z. Shkundin Acoustical Physics, 44, 1, pp. 68-72 (1998)

[11] R. Mittra and S. W. Lee, Analytical Techniques in the Theory of Guided Waves (Macmillan, New York, 1971) 302 p .

[12] S.Z. Shkundin, V.A. Rumyantseva Measurement Techniques 44(1) pp. 87-92 (2001)

[13] Y. Ando Acustica. 22 pp. 219-225 (1969/70)

[14] S.Z. Shkundin, O.A. Kremleva, V.A. Rumyantseva Teoriya akusticheskoj anemometrii [Theory of acoustic anemometry] (Akademy of Mining Science, Moscow, 2001) 240 p. (in Russian)

[15] S.Z. Shkundin, S.I. Buyanov, V.A. Rumyantseva Gorny Informatsionno-Analiticheskiy Byulleten (Nauchno-Teknicheskii Zhurnal) GIAB [Mining Informational and Analytical Bulletin (Scientific and Technical Journal)] 10, pp. 181-182. (2000) (in Russian) 